SCIENTIFIC REPORT

\title{
Outcomes after combination photodynamic therapy and immunosuppression for inflammatory subfoveal choroidal neovascularisation
}

\author{
A Hogan, U Behan, D J Kilmartin
}

Br J Ophthalmol 2005;89:1109-1111. doi: 10.1136/bjo.2004.063024

Aim: To report the visual and angiographic outcomes after combination photodynamic therapy (PDT) and immunosuppression for inflammatory subfoveal choroidal neovascularisation (CNV).

Methods: Retrospective review of six consecutive patients, five female and one male, aged 23-40 years with active subfoveal CNV secondary to posterior uveitis. Patients received either intravitreal triamcinolone or systemic immunosuppression (mycophenolate mofetil, tacrolimus) and PDT. Five patients had intravitreal triamcinolone injections and two patients were on systemic immunosuppression; all patients underwent PDT (mean two treatments). Visual acuity was measured on a 2 metre ETDRS chart and fluorescein angiograms were performed at each visit.

Results: Median follow up was 15 months (range 10-31). Vision improved by a median of 13 letters in five patients and remained stable $( \pm 1$ letter) in one patient. Median visual acuity improved from $20 / 160$ at presentation to $20 / 40$ at latest follow up $(p=0.03)$. There was a reduction in clinical exudation and cessation of angiographic leakage in all six patients. All interventions were well tolerated.

Conclusion: Combination PDT and immunosuppression may be a useful therapeutic option for young patients with active inflammatory subfoveal CNV.

\footnotetext{
C
} horoidal neovascularisation (CNV) in younger patients is a well documented complication of posterior uveitis and white dot syndromes with and without inflammation. Traditional options available for managing these lesions include observation, laser photocoagulation, local and systemic corticosteroids, and surgical removal; all with potential limitations. The natural course of subfoveal CNV is poor if untreated $^{12}$ and laser photocoagulation of subfoveal membranes is unacceptable clinically because of the severe vision loss induced as a result of damage of the neurosensory retina. Corticosteroids and immunosuppressive agents have an established role in treating posterior uveitis, but their role is unclear in treating inflammatory CNV.

More recently some benefit has been demonstrated with photodynamic therapy (PDT) for inflammatory subfoveal $\mathrm{CNV}^{3-5}$ The short term thrombogenic effects of PDT in combination with longer term anti-inflammatory effects of local and systemic immunosuppression may be more successful in achieving closure of these membranes than either treatment alone.

We assessed the potential role of PDT with adjunctive immunosuppression for young patients with inflammatory CNV.

\section{PATIENTS AND METHODS}

Retrospective review was performed of case notes and fluorescein angiograms of six consecutive patients, less than 50 years of age, with active CNV treated with PDT and intravitreal triamcinolone (IVT) or systemic immunosuppression (mycophenolate mofetil, tacrolimus). The diagnoses were presumed ocular histoplasmosis syndrome (POHS) in three patients, punctate inner choroidopathy (PIC) in two patients, and intermediate uveitis in one patient. Four patients had active inflammatory subfoveal CNV alone and two patients (patients 3 and 5) had active choroiditis in addition to subfoveal CNV at presentation.

Active CNV, with documented visual loss, was diagnosed clinically by the presence of subretinal fluid (six eyes) and haemorrhage (two eyes) and confirmed angiographically. Patients who had combination therapy were treated initially with PDT, followed by intravitreal triamcinolone (IVT) within 2 weeks. The intravitreal injections were given later to ensure a clear visual axis for the administration of PDT. Repeat PDT was performed at 3 monthly intervals for any angiographic subfoveal CNV leakage. Repeat intravitreal injections were given when there was persistent serous fluid clinically or persistent angiographic leakage within the 3 month interval precluding repeat PDT. Patient 2, who was 26 weeks pregnant at initial presentation and patient 6 , because of a history of vitritis, received IVT as the first line treatment.

Patient 3 (POHS) and patient 5 (PIC) (fig l) at presentation had active focal choroiditis and subfoveal inflammatory $\mathrm{CNV}$ and were commenced on systemic immunosuppression. Initially they were controlled with mycophenolate mofetil ( $2 \mathrm{~g}$ daily); both required additional tacrolimus ( $2 \mathrm{mg}$ daily) to control recurrent choroiditis. Both have remained on systemic immunosuppression for 31 months and 14 months, respectively.

At each review patients underwent visual acuity assessment on an ETDRS chart, anterior segment examination, tonometry, detailed fundus examination with a biomicroscopic lens, fundus photography, and fluorescein angiography. All patients were reviewed at 1 week and 3 monthly intervals thereafter following PDT.

PDT was administered by the TAP study group protocol. ${ }^{6}$ Intravitreal triamcinolone ( $4 \mathrm{mg}$ in $0.1 \mathrm{ml}$ ) was administered in the operating theatre under local anaesthesia.

We assessed outcomes using number of letters gained on an ETDRS chart at 2 metres and fluorescein angiographic closure of the CNV complex. Any adverse effect of treatment

Abbreviations: CNV, choroidal neovascularisation; IL, interleukin; IVT, intravitreal triamcinolone; PDT, photodynamic therapy; PIC, punctate inner choroidopathy; POHS, presumed ocular histoplasmosis syndrome; TNF, tumour necrosis factor; VOH study, Verteporfin in Ocular Histoplasmosis study 

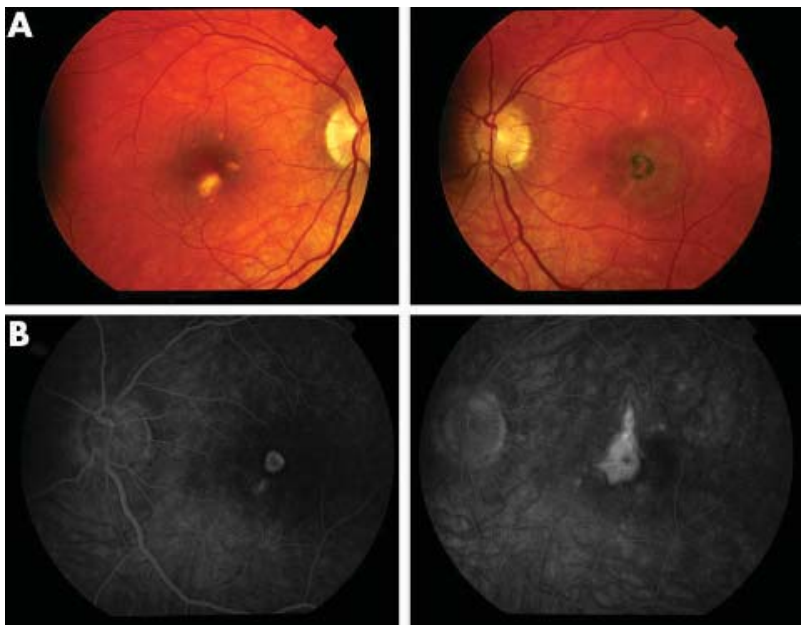

Figure 1 (A) Fundus photographs of patient 5 (PIC) at presentation showing inactive lesions in the posterior pole of both eyes and left subfoveal CNV. Left visual acuity was 20/100. (B) Late frame fluorescein angiogram showing left subfoveal purely classic $\mathrm{CNV}$ at initial presentation (left) and staining only on late frames at latest follow up (right). Visual acuity has improved to $20 / 40$.

was noted. Initial and final visual acuity was compared by conversion to $\log$ MAR using a paired two tailed Student's $t$ test.

\section{RESULTS}

Of the six patients, five were female and one was male. The median age at presentation was 30 years (range 2340 years). The median duration of symptoms was 6 weeks (range 1-20 weeks). All six patients had subfoveal CNV; five patients had purely classic lesions and one patient had a predominantly classic lesion on fluorescein angiogram. Median follow up was 15 months (range 10-31 months). All patients received PDT and the median number of PDT treatments was two (range one to three). Four patients had one, and one patient had two intravitreal triamcinolone injections. Baseline patient and CNV characteristics, treatments, and vision outcomes are summarised in table 1 .

\section{Vision and fluorescein angiographic outcomes}

Vision improved in five (83\%) and remained stable in one $(17 \%)$ of the six patients. These five patients had a median improvement of 13 letters on the logMAR chart (range 7-36), approximately equivalent to three lines. The patient whose vision remained stable had an improvement of one letter on the chart. At presentation median visual acuity was 20/160 (range 20/40-20/250) and at latest follow up was 20/40 (range 20/25-20/250), approximately a six line improvement $(\mathrm{p}=0.03)$.

At presentation median lesion size was $1300 \mu \mathrm{m}$ (range $800-3200 \mu \mathrm{m}$ ) and haemorrhage was present in two eyes, involving fixation in patient 1 . At latest follow up all CNV lesions had minimal (<50\%) fibrosis and no haemorrhage, with absence of angiographic leakage. No significant retinal pigment epithelial changes were noted.

\section{Adverse effects}

No complications were recorded in relation to PDT therapy. No patient developed any complication related to the intravitreal injection; in particular, there were no cases of endophthalmitis, retinal detachment, raised intraocular pressure, or development of cataract. One patient on mycophenolate mofetil reported mild gastrointestinal upset. The haematological and biochemical parameters of patients taking mycophenolate mofetil and tacrolimus remained within normal limits.

\section{DISCUSSION}

Our series appears to demonstrate that combination PDT with intravitreal triamcinolone and/or systemic immunosuppression achieved angiographic closure of the neovascular complex in all patients with concomitant improvement of vision in the majority (83\%) at latest follow up. This compares favourably with the natural history of CNV in younger patients with POHS $^{1}$ and PIC. ${ }^{2}$

Until recently, treatment options for inflammatory $\mathrm{CNV}$ were limited to observation, argon laser photocoagulation, corticosteroids, and surgical removal. Argon laser photocoagulation of subfoveal $\mathrm{CNV}$ is unacceptable owing to the ensuing immediate profound iatrogenic visual loss and the role of corticosteroids alone is not definitive. Flaxel et al demonstrated stabilisation or improvement of vision in 10 of 12 eyes with inflammatory CNV secondary to PIC or multifocal inner choroiditis following steroid therapy alone. Stabilisation of vision at 3 months has also been achieved from the use of steroids for $\mathrm{CNV}$ in a series of 18 patients with POHS $^{8}$; however, other studies have not shown a significant visual improvement following steroids for CNV in $\mathrm{PIC}^{9}$ or multifocal choroiditis and panuveitis (MCP). ${ }^{10}$ Dees et $\mathrm{al}^{11}$ showed a potential benefit from immunosuppression (corticosteroids and/or ciclosporin) for CNV associated with endogenous posterior uveitis, with 53\% stabilising or improving vision and $76 \%$ achieving angiographic CNV closure. Mycophenolate mofetil ${ }^{12}$ and tacrolimus ${ }^{13}$ have proved efficacy in managing refractory posterior uveitis, though there are no reports of their specific use in inflammatory $\mathrm{CNV}$.

Table 1 Patient and CNV characteristics, treatments, and outcomes

\begin{tabular}{|c|c|c|c|c|c|c|c|}
\hline \multirow[b]{2}{*}{ Patient/sex/diagnosis/age (years) } & \multicolumn{2}{|c|}{ Initial fluorescein angiogram } & \multirow[b]{2}{*}{ PDT } & \multirow[b]{2}{*}{$\begin{array}{l}\text { Intravitreal } \\
\text { triamcinolone/other }\end{array}$} & \multicolumn{3}{|c|}{ Vision changes $\dagger$} \\
\hline & Size $^{*}(\mu \mathrm{m})$ & $\%$ blood & & & Initial VA & Final VA & $\begin{array}{l}\text { Change in } \\
\text { lefters }\end{array}$ \\
\hline 1/M/POHS/39 & 3200 & $25-50$ & 3 & 1 & $20 / 250$ & $20 / 250$ & 1 \\
\hline 2/F/POHS/40 & 2400 & 0 & 1 & 1 & $20 / 200$ & $20 / 125$ & 15 \\
\hline 3/F/POHS/29 & 800 & 0 & 3 & $\begin{array}{l}1 \\
\text { MMF, Tacrolimus }\end{array}$ & $20 / 40$ & $20 / 25$ & 7 \\
\hline 4/F/PIC/23 & 970 & $1-24$ & 2 & 1 & $20 / 50$ & $20 / 32$ & 10 \\
\hline $5 / \mathrm{F} / \mathrm{PIC} / 31$ & 1200 & 0 & 2 & MMF, Tacrolimus & $20 / 100$ & $20 / 40$ & 21 \\
\hline $6 / F /$ intermediate uveitis/23 & 1300 & 0 & 1 & 2 & $20 / 200$ & $20 / 40$ & 36 \\
\hline
\end{tabular}

*Size $(\mu \mathrm{m})$ : greatest linear dimension of entire CNV complex (CNV + haemorrhage + elevated blocked fluorescence).

†Vision measured on a logMAR chart. Median initial VA 20/160 and median final VA 20/50. POHS, presumed ocular histoplasmosis syndrome; PIC, punctate inner choroidopathy; PDT, photodynamic therapy; VA, visual acuity. 
Initial studies of the role of PDT in young adults with inflammatory or idiopathic CNV have been promising with more patients achieving stable or improved vision compared to those with age related macular degeneration. ${ }^{34}$ The Verteporfin in Ocular Histoplasmosis (VOH) study group ${ }^{5}$ studied the role of PDT in inflammatory CNV and showed that $45 \%$ improved seven or more letters and $18 \%$ lost eight or more letters at 2 years. No patient in our series lost any letters and the mean change was a 14 letter improvement.

Histopathology of surgically excised neovascular membranes has demonstrated the most common cellular components are vascular endothelium and retinal pigment epithelium ${ }^{14}$ with inflammatory mediators such as macrophages and lymphocytes and proangiogenic factors such as VEGF present. ${ }^{15}$ Oh et $a l^{16}$ suggested that release of interleukin (IL)- $1 \beta$ and tumour necrosis factor (TNF)- $\alpha$ from macrophages stimulates production of VEGF by the retinal pigment epithelium, contributing to the neovascular process. This inflammatory environment, therefore, promotes the development of CNV. PDT has a profound early antiangiogenic effect, mediating intraluminal vascular thrombosis through generation of reactive oxygen intermediates. Furthermore, PDT has some inherent anti-inflammatory properties, mediating lymphocyte apoptosis ${ }^{17}$ and IL-10 secretion, ${ }^{18}$ which may be optimised in an environment of dampened inflammation. The reduced PDT re-treatment rate in this series (two) compared to the $\mathrm{VOH}$ study ${ }^{5}$ may be the result of the combined early anti-inflammatory effect of PDT with the later more sustained effect of immunosuppression.

Triamcinolone acetonide, mycophenolate mofetil, and tacrolimus have proved roles in ocular inflammatory conditions. The use of intravitreal triamcinolone alone for juxtafoveal and subfoveal CNV in a series of 10 patients with POHS has recently been described ${ }^{19} ; 30 \%$ gained five or more letters, 50\% had stable vision, and 20\% lost 5-14 letters over a median follow up of 17 months. Recent interest has focused on the role of combined PDT with intravitreal triamcinolone for age related neovascular membranes, with promising results; $57 \%$ achieving stable or improved vision in a series of 14 patients with a median follow up of 18 months. ${ }^{20}$ With combined treatment in our series, $83 \%$ have improved their vision and no patient lost any letters.

Our visual and fluorescein angiographic outcomes following combination therapy in this series are better than outcomes following PDT alone $e^{3-5}$ or systemic corticosteroids $^{7-10}$ or intravitreal triamcinolone ${ }^{19}$ alone. In addition, combining PDT with immunosuppression may reduce the number of re-treatments needed. Combination therapy in this series of patients was well tolerated and no complications were recorded. The weaknesses of this study include the small numbers of patients, the relatively short follow up time, and its retrospective nature. In addition, not all patients received the same treatments or in the same temporal sequence. Further randomised controlled trials are necessary to elucidate the role of PDT in combination with immunosuppression, whether systemic or local, compared with PDT alone.

\section{Authors' affiliation}

A Hogan, Research Foundation, Royal Victoria Eye and Ear Hospital, Adelaide Road, Dublin 2, Ireland
U Behan, Department of Ophthalmology, Royal Victoria Eye and Ear Hospital, Adelaide Road, Dublin 2, Ireland

D J Kilmartin, Department of Ophthalmology, Royal College of Surgeons in Ireland, Royal Victoria Eye and Ear Hospital, Adelaide Road, Dublin 2, Ireland

Competing interests: none declared

Correspondence to: Mr Dara J Kilmartin, MSc, FRCSI, FRCOphth, Department of Ophthalmology, Royal College of Surgeons in Ireland, Royal Victoria Eye and Ear Hospital, Adelaide Road, Dublin 2, Ireland; dikilmartin@eircom.net

Accepted for publication 9 February 2005

\section{REFERENCES}

1 Macular Photocoagulation Study Group. Krypton laser photocoagulation for neovascular lesions of ocular histoplasmosis. Results of a randomized clinical neovascular lesions of ocular histoplasmosis. Rest
trial. Arch Ophthalmol 1987; 105:1499-507.

2 Watske RC, Packer AJ, Folk JC, et al. Punctate inner choroidopathy. Am J Ophthalmol 1984;98:572-84.

3 Rogers AH, Duker JS, Nichols N, et al. Photodynamic therapy of idiopathic and inflammatory choroidal neovascularization in young adults. Ophthalmology 2003;110:1315-20.

4 Wachtlin J, Heimann $\mathrm{H}$, Behme $\mathrm{T}$, et al. Long-term results after photodynamic therapy with verteporfin for choroidal neovascularization secondary to inflammatory chorioretinal diseases. Graefes Arch Clin Exp Ophthalmol 2003;241:899-906

5 Rosenfeld PJ, Saperstein DA, Bressler NM, et al. Verteporfin in Ocular Histoplasmosis Study Group. Photodynamic therapy with verteporfin in ocular histoplasmosis: uncontrolled, open-label-2-year study, Ophthalmology 2004; 111:1725-33

6 Treatment of Age-related Macular Degeneration with Photodynamic Therapy (TAP) Study Group. Photodynamic therapy of subfoveal choroidal neovascularization in age-related macular degeneration with verteporfin. One-year results of 2 randomized clinical trials - TAP Report 1. Arch Ophthalmol 1999; 117:1329-45.

7 Flaxel CJ, Owens SL, Mulholland B, et al. The use of corticosteroids for choroidal neovascularization in young patients. Eye 1998;12:266-72.

8 Martidis A, Miller DG, Ciulla TG, et al. Corticosteroids as an antiangiogenic agent for Histoplasmosis-related subfoveal choroidal neovascularization. J Ocul Pharmacol Ther 1999;15:425-8.

9 Olsen TW, Capone A Jr, Sternberg P Jr, et al. ubfoveal choroidal neovascularization in punctuate inner choroidopathy. Surgical management and pathologic findings. Ophthalmology 1996;103:2061-9.

10 Spaide RF, Freund KB, Slakter J, et al. Treatment of subfoveal choroidal neovascularization associated with multifocal choroiditis and panuveitis with photodynamic therapy. Retina 2002;22:545-9.

11 Dees C, Arnold JJ, Forrester JV, et al. Immunosuppressive treatment of choroidal neovascularization associated with endogenous posterior uveitis. Arch Ophthalmol 1998;116:1456-61.

12 Kilmartin DJ, Forrester JV, Dick AD. Rescue therapy with mycophenolate mofetil in refractory uveitis. Lancet 1998;352:25-6

13 Kilmartin DJ, Forrester JV, Dick AD. Tacrolimus (FK506) in failed cyclosporine A therapy in endogenous posterior uveitis. Ocul immunol Inflamm 1998;6:101-9.

14 Groosniklaus HE, Green WR. Histopatholgic and ultrastructural findings of surgically excised choroidal neovascularization. Arch Ophthalmol 1998; 116:745-9.

15 Kwak N, Okamoto N, Wood JM, et al. VEGF is major stimulator in model of choroidal neovascularization. Invest Ophthalmol Vis Sci 2000;41:3158-64.

16 Oh H, Takagi H, Takagi C, et al. The potential angiogenic role of macrophages in the formation of choroidal neovascular membranes. Invest Ophthalmol Vis Sci 1999;40:1891-1898.

17 Hunt DW, Jiang H, Granville DJ, et al. Consequences of photodynamic treatment of resting and activated peripheral T lymphocytes. Immunopharmacology 1999;41:31-44.

18 Simkin GO, Tao JS, Levy LG, et al. IL-10 contributes to the inhibition of contact hypersensitivity in mice treated with photodynamic therapy. J Immunol 2000;164:2457-62.

19 Rechtman E, Allen VD, Danis RP, et al. Intravitreal triamcinolone for choroidal neovascularization in ocular histoplasmosis syndrome. Am J Ophthalmol 2003;136(4):739-41.

20 Rechtman E, Danis RP, Pratt LM, et al. Intravitreal triamcinolone with photodynamic therapy for subfoveal choroidal neovascularization in age related macular degeneration. Br J Ophthalmol 2004;88:344-7. 